\title{
Dynamic Downlink Spectrum Access for D2D-Enabled Heterogeneous Networks
}

\author{
Redha M. Radaydeh*, Fawaz S. Al-Qahtani ${ }^{\dagger}$, Abdulkadir Celik*, and Mohamed-Slim Alouini* \\ ${ }^{*}$ Computer, Electrical, and Mathematical Sciences and Engineering Division \\ King Abdullah University of Science and Technology (KAUST), Thuwal, 23955-6900, KSA \\ ${ }^{\dagger}$ Department of Electrical and Computer Engineering, Texas A\&M University, Doha, Qatar
}

\begin{abstract}
This paper proposes new approaches for underlay device-to-device (D2D) communication in spectrum-shared heterogeneous cellular networks. It considers devices that share downlink resources and have an enabled D2D feature to improve coverage. The mode of operation classifies devices according to their experienced base station (BS) coverage, potential to be served by BS, ability of BS to meet their quality of service $(\mathrm{QoS})$, and their downlink resources occupancy. The initiation of D2D cooperation is conditioned on proposed provisional access by an active device, wherein its serving BS attempts to meet its QoS using as low number of spectrum channels as possible, while treating remaining channels for feasible D2D cooperation. Detailed formulations for the mode of operation and a proposed D2D path allocation scheme are presented under perfect and imperfect operation scenarios. The developed results are generally applicable for any performance metric and network model.
\end{abstract}

\section{INTRODUCTION}

Device-to-Device (D2D) communication has been considered as a promising scheme in future generations of cellular networks [1]. It can be operated either on licensed cellular spectrum (i.e., in-band) or on unlicensed spectrum (i.e., outband), where the latter can be subject to uncontrolled interference. D2D technology can offload traffic from the serving $\mathrm{BS}$, thereby reducing the centralized processing as well as decreasing energy consumption of the serving BS [2], [3]. However, there are many technical challenges that need to be tackled to ensure a successful operation of D2D technology within future cellular architectures.

Among the technical challenges that face D2D communication in cellular networks are the need for improved interference management, efficient power control, mode selection, and sophisticated allocation of spectrum resources [3]. The issue of resource allocation and interference mitigation has been addressed between cellular paths and D2D paths, cellular paths alone, or D2D paths alone. For instance, with full knowledge channel state information (CSI) of all cellular links at the serving BS, the cellular-to-D2D interference in downlink may be managed using transmit beamforming [4]. The works in [5]-[6] proposed interference-limited schemes to manage interference from cellular paths to D2D path, while meeting certain quality targets. In addition, different algorithms for resources distribution and/or optimization for D2D communication have been addressed for various objectives, wherein the adopted approaches for solutions range from basic models [8] to complicated models [9], [10].
A part from complicated formulations that demands unique processing load, and different from the previous works that utilize applied mathematical theories to analyze resource or interference management in cellular networks with D2D communication, this paper develops new approaches that address many practical constraints and allow for the concurrent operation of existing cellular users and an underlay D2D system on the licensed downlink. The developed approaches herein jointly treat the issues of devices mode selection, interference mitigation, and resource allocation for underlay D2D communication. It takes into consideration that an authorized cellular device attempts to receive service from its serving $\mathrm{BS}$, and that the establishment of D2D cooperation is conditioned on active devices condition. Herein, the D2D service is adaptively managed such that the served device by the BS attempts to first meet its required QoS, and then it can utilize the remaining unused spectrum bands (channels) to serve other active device(s), which can not be served by BS, through direct D2D link(s). Capitalizing on the findings in [11], which investigated the impact of user identities and their access conditions in the context of closed small-cell networks, and the improved interference-free resource allocation proposals for coordinated multiuser open-access small cells in [12], various operational and design aspects for a suitable D2D cooperation path that can meet specific design targets at the served device are then developed and quantified.

The rest of the paper is organized as follows. Section II presents the system model and mode of operation. Section III describes the classes of devices and their associated channels. Section IV presents the scheme for D2D path identification under perfect and imperfect operations. Section V discusses selected numerical results, and Section VI contains the concluding remarks.

\section{System Model And Mode of Operation}

\section{A. Overview of System Model}

The system model under consideration treats a macrocell coverage area as part of a cellular system. Active users devices are unconditionally authorized to access licensed spectrum resources, which can be in the form of physical channels as in orthogonal-frequency division multiplexing (OFDM) system. Moreover, to overcome the occasions of BS service interruption or macrocell poor coverage, authorized devices are allowed to initiate D2D cooperation whenever these links are 


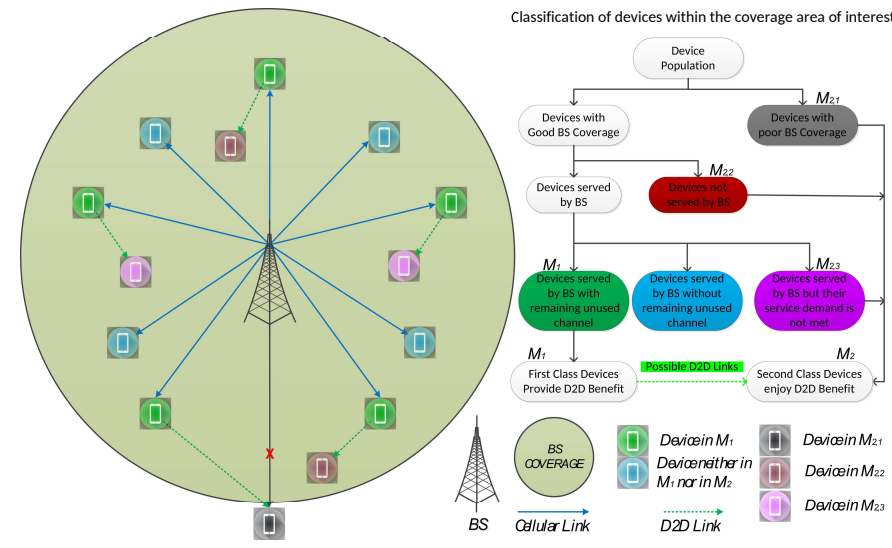

Figure 1. Classes of devices for potential D2D communication in the macrocell coverage area.

realizable. These D2D links can be established using the same resources that are available to the serving BS. The possible D2D links between devices may be suitable to provide service to devices whose QoS demand from the BS undergoes deep degradation or they are not permitted to access BS resources at a particular time. Moreover, they are established to transfer data between devices without the need to overload BS.

The coverage area of the macrocell contains a variable number of $M$ authorized devices, which can be randomly distributed inside the macrocell coverage space according to a 2-D point distribution, such as Poisson point field ${ }^{1}$. The devices can potentially be assigned any of the downlink cellular resources by the serving BS. The number of them, denoted by $N$, is assumed to be known to all authorized nodes.

\section{B. Mode of Operation}

During a particular service time, the authorized active devices cooperate with their serving BS to meet their individual QoS demands. Accordingly, these devices can be classified into two groups, as shown in Fig. 1. The first group contains devices that are in the range of good BS coverage, whereas the second group contains devices that experience poor BS coverage.

Due to constraints that can be imposed on the number of supported devices and/or the maximum data rate that can be transferred by the serving BS during a service time, some of devices that experience good BS coverage may not be granted service by BS. Moreover, other devices that experience good BS coverage and permitted to access BS resources are served to use a minimal number of channels to meet their individual QoS demands. In the later case, if the BS meets a device QoS demand, the device is permitted to treat its unused channels to serve D2D communication. This approach can facilitate the processing load at devices since the engagement with

\footnotetext{
${ }^{1}$ The spatial locations of advices can be effectively captured through largescale fading models associated with channel gains, which count for relative wave propagation separations between transceivers.
}

the serving BS and the support of D2D cooperation can be managed on orthogonal domains.

An active device will not support D2D cooperation if the BS can not meet its downlink QoS demand or if the BS needs to use all available channels to satisfy its QoS demand. Therefore, a device that is fully served by BS will have a variable number of channels that can exploited for D2D communication.

The D2D mode discovery operation starts from a device whose QoS can be met by BS, which belongs to the first class of devices, as in Fig. 1. This device broadcasts a control message within its D2D coverage to indicate that it can serve other devices that are not served by BS. A device whose QoS is not met through the serving BS can be in the groups of devices that either experience poor BS coverage, whose request for service are blocked at the serving BS, or whose QoS limit is not met even with the use of all channels, which represent the second class of devices in Fig. 1. Such a device then broadcasts a control message to request a D2D service. Then, a device from the second class will wait for acknowledgment (ACK) response(s) from any close device(s) from the first class to confirm the availability for D2D cooperation.

If a device from the second class does not receive any ACK massage, it has to wait for a new service time and restart the search for downlink service by first coordinating with the serving BS. On the other hand, if it receives at least one ACK message, it starts to collect channel state information (CSI) associated with feasible device(s) from the first class. It will be used to identify the most suitable device and its associated channel to establish a desired D2D path according to the adopted D2D path identification scheme.

\section{DEVICES AND CHANNELS FOR D2D COOPERATION}

This Section develops precise models to define the sets of devices in the two classes described above. Moreover, the set of unused channels per an active device from the first class, which can potentially support D2D cooperation is quantified.

\section{A. Classes of Active Devices}

Based on the discussions in subsection II-B, let $\mathcal{M}_{1}$ and $\mathcal{M}_{2}$ be disjoint sets, which contain the indexes of devices that belong to the first class and second class of devices, respectively. It follows that $M \geq\left|\mathcal{M}_{1}\right|+\left|\mathcal{M}_{2}\right|$. Moreover, the devices whose indexes in $\mathcal{M}_{2}$ are partitioned into three disjoint sets, namely $\mathcal{M}_{2}=\mathcal{M}_{2,1} \cup \mathcal{M}_{2,2} \cup \mathcal{M}_{2,3}$. The subset $\mathcal{M}_{2,1}$ contains indexes of devices that undergo poor coverage of the serving $\mathrm{BS}, \mathcal{M}_{2,2}$ contains devices whose service requests to the serving BS are blocked, and $\mathcal{M}_{2,3}$ contains devices whose QoS demands are not met by the serving BS even when all channels are used. The following discussions are divided into two subparts, which explain operation conditions of devices in $\mathcal{M}_{1}$ and $\mathcal{M}_{2}$, respectively.

1) Devices in $\mathcal{M}_{1}$ : To have $m_{1} \in \mathcal{M}_{1}$, the $m_{1}$ th device has to meet several conditions. First, it should be within the good coverage of BS. Second, it has to be one the devices whose downlink service request is not blocked by BS. Third, its QoS demand can be met through downlink service from BS. And 
finally, it has unused channels that can be utilized to support D2D cooperation. Therefore, after utilizing the independence between the preceding conditions, the likelihood of $m_{1} \in \mathcal{M}_{1}$ can be expressed as

$$
\begin{aligned}
& \operatorname{Pr}\left\{m_{1} \in \mathcal{M}_{1}\right\}=\operatorname{Pr}\left\{\widetilde{s}_{m_{1}}>\widetilde{s}_{\text {min }}\right\}\left(1-p_{\text {block }}\right) \\
& \times \operatorname{Pr}\left\{\bar{C}_{m_{1}} \geq C_{\text {th }, m_{1}} \& 1 \leq N_{m_{1}} \leq N-1\right\},
\end{aligned}
$$

where $\widetilde{s}_{m_{1}} \triangleq P_{t, 0}\left|h_{m_{1}, 0}\right|^{2} d_{m_{1}, 0}^{-\alpha}$ is the received coverage power level that is observed at the $m_{1}$ th device from the serving $\mathrm{BS}$, in which $P_{t, 0}$ is an average coverage signaling power that is broadcasted by the serving $\mathrm{BS}, h_{m_{1}, 0}$ is a complex-valued Gaussian random variable, and it represents the channel gain of the link between the BS and the $m_{1}$ th device, $\left|h_{m_{1}, 0}\right|^{2}$ follows an exponential distribution of mean value $\mathbb{E}\left\{\left|h_{m_{1}, 0}\right|^{2}\right\}=\Omega_{m_{1}, 0}$, where $\mathbb{E}\{\cdot\}$ refers to the statistical expectation of the quantity between brackets. Moreover, the term $d_{m_{1}, 0}$ is the spatial distance between the BS and the $m_{1}$ th device, and $\alpha$ is the associated path loss exponent. Moreover, the term $\widetilde{s}_{\min }$ in (1) is a design parameter that represents the minimal threshold of $\widetilde{s}_{m_{1}}$ to be within an acceptable coverage range of BS coverage. Moreover, the term $p_{\text {block }}$ refers to the service blockage probability when the $m_{1}$ th device attempts to request downlink service from $\mathrm{BS}$, which is related to the adopted traffic model. The term $N_{m_{1}}$ in (1) refers to the number of used channels by the $m_{1}$ th device, and $\bar{C}_{m_{1}}$ is defined as

$$
\bar{C}_{m_{1}} \triangleq \sum_{n=1}^{N_{m_{1}}} C_{m_{1}, n}=\sum_{n=1}^{N_{m_{1}}} \log _{2}\left(1+\gamma_{m_{1}, n}\right),
$$

which represents the aggregate capacity at the $m_{1}$ th device (in bit/s/Hz), and $C_{\mathrm{th}, m_{1}}$ represents the capacity threshold (i.e., QoS demand) at the $m_{1}$ th device. The term $\gamma_{m_{1}, n}$ refers to the received SINR at the $m_{1}$ th device from the serving BS over the over $n$th channel, which can be defined as

$$
\gamma_{m_{1}, n}=\frac{s_{D, m_{1}, n}}{s_{I, m_{1}, n}+\sigma^{2}},
$$

where $s_{D, m_{1}, n} \triangleq P_{t}\left|h_{D, m_{1}, n}\right|^{2} d_{m_{1}, 0}^{-\alpha}$ is the received desired power at the $m_{1}$ th device on the $n$th channel, in which $P_{t}$ refers to the average transmit power of data symbol, and $h_{D, m_{1}, n}$ is the associated channel gain with $\left|h_{D, m_{1}, n}\right|^{2}$ follows an exponential distribution of an average value $\mathbb{E}\left\{\left|h_{D, m_{1}, n}\right|^{2}\right\}=\Omega_{D, m_{1}, n}$. In (3), the term $s_{I, m_{1}, n}=\sum_{j=1}^{K} P_{t}\left|h_{I, j, m_{1}, n}\right|^{2} d_{I, j, m_{1}}^{-\alpha_{I, j}}$ is the aggregate interference power that is observed at the $m_{1}$ th device on the $n$th channel, which is a superposition of interference powers observed due to the concurrent service that are granted to $K$ nearby active devices on the same $n$th channel, wherein $\left\{\left|h_{I, j, m_{1}, n}\right|^{2}\right\}_{j=1}^{K}$ are statistically independent exponentially distributed random variables of mean values $\mathbb{E}\left\{\left|h_{I, j, m_{1}, n}\right|^{2}\right\}=\Omega_{I, j, m_{1}, n^{2}}$, and $\sigma^{2}$ is the average power

\footnotetext{
${ }^{2}$ It is worth mentioning that the developments in this paper are broadly applicable for any channel gains models for the desired link as well as interference sources. The models described herein are used as examples for tractable treatment.
}

of the background white noise. The statistics of $\gamma_{m_{1}, n}$ can be obtained as

$$
\begin{aligned}
& \operatorname{Pr}\left\{\gamma_{m_{1}, n}<x\right\} \\
& =\mathbb{E}_{s_{I, m_{1}, n}}\left\{\operatorname{Pr}\left\{s_{D, m_{1}, n}<x\left(y+\sigma^{2}\right) \mid s_{I, m_{1}, n}=y\right\}\right\} .
\end{aligned}
$$

The last term in (1) can be further quantified as follows

$$
\begin{aligned}
& \operatorname{Pr}\left\{\bar{C}_{m_{1}} \geq C_{\mathrm{th}, m_{1}} \& 1 \leq N_{m_{1}} \leq N-1\right\} \\
& =\sum_{n=1}^{N-1} \operatorname{Pr}\left\{\bar{C}_{m_{1}} \geq C_{\mathrm{th}, m_{1}} \mid N_{m_{1}}=n\right\} \operatorname{Pr}\left\{N_{m_{1}}=n\right\} .
\end{aligned}
$$

Note that the device that satisfies (5) will have its index belongs to $\mathcal{M}_{1}$ as long as the number of used channels is below $N$, which are the cases described above. If the device needs to use all channels in order to meet its QoS demand, then it will not belong to $\mathcal{M}_{1}$ (nor to $\mathcal{M}_{2}$, as will be discussed below) as it will have no remaining channels to support D2D cooperation but has its QoS demand be satisfied from the serving BS using all available channels.

2) Devices in $\mathcal{M}_{2}$ : The device whose index belongs to the set $\mathcal{M}_{2}=\mathcal{M}_{2,1} \cup \mathcal{M}_{2,2} \cup \mathcal{M}_{2,3}$ (as stated above), say the $m_{2}$ th device, has different likelihoods to be in $\mathcal{M}_{2}$. Specifically, for indexes of devices that belong to the subset $\mathcal{M}_{2,1}$, the likelihood that $m_{2} \in \mathcal{M}_{2,1}$ can be expressed as

$$
\operatorname{Pr}\left\{m_{2} \in \mathcal{M}_{2,1}\right\}=\operatorname{Pr}\left\{\widetilde{s}_{m_{2}}<\widetilde{s}_{\min }\right\},
$$

which refers to the device that receives a coverage signaling power below the minimal required limit, $\widetilde{s}_{\text {min }}$, to be within a good coverage of BS. Moreover, the likelihood that $m_{2} \in \mathcal{M}_{2,2}$, which models the case when the $m_{2}$ device experiences acceptable BS coverage but it is blocked from receiving service from $\mathrm{BS}$, can be expressed as

$$
\operatorname{Pr}\left\{m_{2} \in \mathcal{M}_{2,2}\right\}=\operatorname{Pr}\left\{\widetilde{s}_{m_{2}}>\widetilde{s}_{\text {min }}\right\} p_{\text {block }}
$$

Finally, the likelihood that $m_{2} \in \mathcal{M}_{2,3}$, which characterizes the case that the $m_{2}$ th device experiences acceptable coverage from the serving BS, its service request is not blocked by the $\mathrm{BS}$, but its requested data rate can not be met even when all channels are used, can be expressed as

$$
\begin{aligned}
& \operatorname{Pr}\left\{m_{2} \in \mathcal{M}_{2,3}\right\} \\
& =\operatorname{Pr}\left\{\widetilde{s}_{m_{2}}>\widetilde{s}_{\text {min }}\right\}\left(1-p_{\text {block }}\right) \operatorname{Pr}\left\{\bar{C}_{m_{2}}<C_{\text {th }, m_{2}} \& N_{m_{2}}=N\right\},
\end{aligned}
$$

where the last term to the right of the equity sign in (8) can be quantified as

$$
\begin{aligned}
& \operatorname{Pr}\left\{\bar{C}_{m_{2}}<C_{\text {th }, m_{2}} \& N_{m_{2}}=N\right\} \\
& =\operatorname{Pr}\left\{\sum_{n=1}^{N} C_{m_{2}, n}<C_{\text {th }, m_{2}}\right\} \operatorname{Pr}\left\{N_{m_{2}}=N\right\} .
\end{aligned}
$$

\section{B. Establishing D2D Path}

For the $j$ th device of interest from $\mathcal{M}_{2}$, it will ideally observe a number of $\left|\mathcal{M}_{1}\right|$ devices that can support its request for $\mathrm{D} 2 \mathrm{D}$ communication. However, the success rate for a device from from $\mathcal{M}_{1}$ for D2D support has to consider its 
ability the to transfer ACK message to the $j$ th device, thereby indicating its readiness for D2D cooperation.

Let $\mathcal{M}_{1, j} \subset \mathcal{M}_{1}$ be a subset of devices that are ready to support D2D cooperation with the $j$ th device, it follows that

$$
\begin{aligned}
p_{m_{1}, j} & =\operatorname{Pr}\left\{m_{1} \in \mathcal{M}_{1} \& \mathrm{ACK} \text { is successful }\right\} \\
& =\operatorname{Pr}\left\{m_{1} \in \mathcal{M}_{1}\right\} p_{\mathrm{ACK}, m_{1}, j}
\end{aligned}
$$

where $p_{\mathrm{ACK}, m_{1}, j}$ refers to the likelihood that ACK message is delivered successfully to the $j$ th device. The statistics of $\left|\mathcal{M}_{1, j}\right|$ can be now expressed as

$$
\operatorname{Pr}\left\{\left|\mathcal{M}_{1, j}\right|=m_{0}\right\} \simeq \frac{e^{-\lambda} \lambda^{m_{0}}}{m_{0} !},
$$

which provides a tight result with $\lambda \triangleq \sum_{m_{1}=1}^{M} p_{m_{1}, j}$.

\section{Channels at Served Devices}

For a device that belongs to $\mathcal{M}_{1, j} \subset \mathcal{M}_{1}$, say the $m_{1}$ th device, the unused channels after the establishment of an acceptable downlink QoS with the serving BS, which is denoted by $L_{m}=N-N_{m_{1}}$, can take values on $\{0,1,2, \ldots, N-1\}$. However, the event $L_{m_{1}}=0$ indicates that the device can not be in $\mathcal{M}_{1}$ (nor in $\mathcal{M}_{1, j}$ ) because it can not support D2D cooperation (i.e., number of used channels is $N_{m}=N$ ). Moreover, the event $L_{m}=N$ is not applicable since it indicates that the device is not active (i.e., number of used channels for BS service is $N_{m}=0$ ). Let the set $\mathcal{N}_{m}$ be the indexes of remaining channels that can be used at the $m_{1}$ th device, whose index belongs to $\mathcal{M}_{1, j}$, to support D2D cooperation, where $\left|\mathcal{N}_{m}\right| \triangleq L_{m}$.

The process starts with the first channel that can be chosen arbitrary (requires no pre-processing or channel monitoring) or based on large-scale channel quality of channels (require longterm prediction of channel conditions). If it is found that the supported capacity on that channel $C_{m, 1}$ is above the requested demand $C_{\mathrm{th}, m}$, the process will be terminated and the number of unused channels becomes $L_{m}=N-1$. Otherwise, an additional channel is exploited to improve the supported QoS, and if the resulting aggregate capacity $\bar{C}_{m}=C_{m, 1}+C_{m, 2}$ is above $C_{\mathrm{th}, m}$ while $C_{m, 1}<C_{\mathrm{th}, m}$, the process will be terminated and the resulting number of unused channels will be $L_{m}=N-2$.

At the $n$th processing stage, for $n=3 \ldots, N-1$, a total of $n$ channels are needed to meet the QoS demand (i.e., $\left.\sum_{r=1}^{n} C_{m, r}>C_{\mathrm{th}, m}\right)$ provided that $\sum_{r=1}^{n-1} C_{m, r}<C_{\mathrm{th}, m}$, and hence, the number of unused channels will be $L_{m}=N-n$. If it is found that the aggregate capacity after the $(N-1)$ st stage does not the required threshold (i.e., $\sum_{r=1}^{N-1} C_{m, r}<C_{\mathrm{th}, m}$ ), the BS attempts to meet the device QoS demand by exploiting the last available channel. In this case, this device index can not be in set $\mathcal{M}_{1}$ because $L_{m}=0$, and hence, it can not support D2D cooperation (i.e., number of used channels is $\left.N_{m}=N\right)$. Moreover, it can neither be in set $\mathcal{M}_{2}$ even if the aggregate capacity is met with the use of all channels (i.e., case of $\sum_{r=1}^{N} C_{m, r}>C_{\text {th }, m}$ while $\left.\sum_{r=1}^{N-1} C_{m, r}<C_{\text {th }, m}\right)$. However, the index of the device will be classified into subset $\mathcal{M}_{2,3}$ if it is found that the aggregate capacity is not met even with the use of all channels (i.e., case of $\sum_{r=1}^{N-1} C_{m, r}<C_{\mathrm{th}, m}$ ).

The statistics of $\left|\mathcal{N}_{m}\right|$ at the $m$ th device can now be expressed as

$$
\operatorname{Pr}\left\{\left|\mathcal{N}_{m}\right|=n\right\}=\operatorname{Pr}\left\{N_{m}=N-n\right\},
$$

wherein

$$
\begin{aligned}
& \operatorname{Pr}\left\{\left|\mathcal{N}_{m}\right|=n\right\} \\
& =\left\{\begin{array}{cl}
\operatorname{Pr}\left\{\sum_{r=1}^{N-1} C_{m, r}<C_{\mathrm{th}, m}\right\}, & n=0 \\
\operatorname{Pr}\left\{\sum_{r=1}^{N-n-1} C_{m, r}<C_{\mathrm{th}, m}\right. & \\
\left.\& \sum_{r=1}^{N-n} C_{m, r} \geq C_{\mathrm{th}, m}\right\}, & 0<n<N-1 \\
\operatorname{Pr}\left\{\gamma_{m, 1} \geq 2^{C_{\mathrm{th}, m}}-1\right\}, & N-1
\end{array}\right.
\end{aligned}
$$

Note that the set of remaining physical channels at the $m_{1}$ th device from the set $\mathcal{M}_{1, j}$ as seen by $j$ th device from the set $\mathcal{M}_{2}$ is determined according to the $m_{1}$ th device channels availability since the $j$ th device can only wait for receiving data through D2D links, and hence, all its channels are treated as unused channels. Therefore, $\mathcal{N}_{m}$ is independent of the index of the potential device to be served through D2D links (i.e., the $j$ th device).

The developed results above will be utilized in the following Section, which treats the proposed scheme for a suitable D2D path identification.

\section{JOINT INTERFERENCE Mitigation AND DESIRED LINK IMPROVEMENT}

This section presents a scheme that can benefit from the availability of multiple devices in the set $\mathcal{M}_{1, j}$ (if any) to reduce the effect of interference while exploiting the channels associated with the identified device (if any) to maximize the received desired power at the served device. The analysis covers perfect and imperfect operations in two separate subsections below.

\section{A. Perfect Path Identification}

For the devices whose indexes are in $\mathcal{M}_{1, j}$, and for given $\left|\mathcal{M}_{1, j}\right|=m_{0}$, define $s_{I,\left(m_{0}\right), n, j} \geq s_{I,\left(m_{0}-1\right), k, j} \geq$ $\cdots \geq s_{I,(1), n, j}$ that is obtained by arranging $\left\{s_{I, m, n, j}\right\}_{m=1}^{m_{0}}$ according to their strengths. It follows that $s_{I,(1), n, j}=$ $\min \left\{s_{I, 1, n, j}, s_{I, 2, n, j}, \ldots, s_{I, m_{0}, n, j}\right\}$. The identification of suitable channel will be performed based on the set $\mathcal{N}_{(1)}$, which includes the indexes of unused channels by the identified device. It aims to maximize the desired power level at the served $j$ th device. Hence, for given $\left|\mathcal{N}_{(1)}\right|=n_{(1)}$, and based on $s_{D,(1),\left(n_{(1)}\right), j} \geq$ $s_{D,(1),\left(n_{(1)}-1\right), j} \geq \cdots \geq s_{D,(1),(1), j}$, it can be written that $s_{D,(1),\left(n_{(1)}\right), j}=\max \left\{s_{D,(1), 1, j}, s_{D,(1), 2, j}, \ldots, s_{D,(1), n_{(1)}, j}\right\}$. The received SINR at the $j$ th device can now be expressed as

$$
\gamma_{\mathrm{SINR}, j}=\frac{s_{D,(1),\left(n_{(1)}\right), j}}{s_{I,(1),\left(n_{(1)}\right), j}+\sigma^{2}} .
$$

Note that $s_{D,(1),\left(n_{(1)}\right), j}$ does not achieve any gain from the advice identification. Likewise, $s_{I,(1),\left(n_{(1)}\right), j}$ does not benefit 
from the desired power improvement through channel identification. The statistics of $\gamma_{\mathrm{SINR}, j}$ is this case can be expressed as

$$
\begin{aligned}
& \operatorname{Pr}\left\{\gamma_{\mathrm{SINR}, j}<x|| \mathcal{M}_{1, j}\left|=m_{0},\right| \mathcal{N}_{(1)} \mid=n_{(1)}\right\} \\
& =\mathbb{E}\left\{\operatorname{Pr}\left\{s_{D,(1),\left(n_{(1)}\right), j}<x\left(y+\sigma^{2}\right) \mid s_{I,(1),\left(n_{(1)}\right), j}=y\right\}\right\},
\end{aligned}
$$

where the term $\operatorname{Pr}\left\{s_{D,(1),\left(n_{(1)}\right), j}<t\right\}$ in (14) can be obtained as

$$
\operatorname{Pr}\left\{s_{D,(1),\left(n_{(1)}\right), j}<t\right\}=\prod_{n^{\prime}=1}^{n_{(1)}} \operatorname{Pr}\left\{s_{D,(1), n^{\prime}, j}<t\right\} .
$$

In addition, the distribution of $s_{I,(1),\left(n_{(1)}\right), j}$ for given $\left|\mathcal{M}_{1, j}\right|=$ $m_{0}$ and $\left|\mathcal{N}_{(1)}\right|=n_{(1)}$, which is needed to compute the expectation operator in (14), can be expressed as

$$
\begin{aligned}
f_{\left.s_{I,(1),(n(1)}\right), j}(t) & =\sum_{m^{\prime \prime}=1}^{m_{0}}\left[\frac{d}{d t} \operatorname{Pr}\left\{s_{I, m^{\prime \prime},\left(n_{(1)}\right), j}<t\right\}\right] \\
& \times \prod_{m^{\prime} \neq m^{\prime \prime}}^{\prod_{m^{\prime}=1}^{m^{\prime}}}\left[1-\operatorname{Pr}\left\{s_{I, m^{\prime},\left(n_{(1)}\right), j}<t\right\}\right]
\end{aligned}
$$

Using the results in (15) and (16) into (14), the term $\operatorname{Pr}\left\{\gamma_{\text {SINR }, j}<x|| \mathcal{M}_{1, j}\left|=m_{0},\right| \mathcal{N}_{(1)} \mid=n_{(1)}\right\}$ can be quantified. Under the special case that all possible D2D paths are identically distributed, it can be obtained that

$$
\begin{aligned}
& \operatorname{Pr}\left\{\gamma_{\mathrm{SINR}, j}<x|| \mathcal{M}_{1, j}\left|=m_{0},\right| \mathcal{N}_{(1)} \mid=n_{(1)}\right\} \\
& =m_{0} \int_{0}^{+\infty}\left[\operatorname{Pr}\left\{s_{D,(1), n, j}<\left(t+\sigma^{2}\right) x\right\}\right]^{n_{(1)}} \\
& \times\left[1-\operatorname{Pr}\left\{s_{I, m,\left(n_{(1)}\right), j}<t\right\}\right]^{m_{0}-1}\left[\frac{d}{d t} \operatorname{Pr}\left\{s_{I, m,\left(n_{(1)}\right), j}<t\right\}\right]
\end{aligned}
$$

The unconditional statistics of $\gamma_{\mathrm{SINR}, j}$ can be then obtained as

$$
\begin{aligned}
& \operatorname{Pr}\left\{\gamma_{\text {SINR }, j}<x\right\} \\
& =\frac{1}{1-\left[\operatorname{Pr}\left\{\left|\mathcal{M}_{1, j}\right|=0\right\}+\operatorname{Pr}\left\{\left|\mathcal{M}_{1, j}\right|=M\right\}\right]} \\
& \times \sum_{m_{0}=1}^{M-1} \frac{1}{1-\operatorname{Pr}\left\{\left|\mathcal{N}_{(1)}\right|=0|| \mathcal{M}_{1, j} \mid=m_{0}\right\}} \\
& \times \sum_{n_{(1)}=1}^{N-1} \operatorname{Pr}\left\{\gamma_{\text {SINR }, j}<x|| \mathcal{M}_{1, j}\left|=m_{0},\right| \mathcal{N}_{(1)} \mid=n_{(1)}\right\} \\
& \times \operatorname{Pr}\left\{\left|\mathcal{N}_{(1)}\right|=n_{(1)}|| \mathcal{M}_{1, j} \mid=m_{0}\right\} \operatorname{Pr}\left\{\left|\mathcal{M}_{1, j}\right|=m_{0}\right\},
\end{aligned}
$$

where the conditional statistics of $\gamma_{\mathrm{SINR}, j}$ in this case are given in (14) or (17). The preceding results include two important cases as limiting scenarios. Specifically, the first case is when the available devices and their associated unused channels are used to improve the desired power level at the served device, whereas the second case is when the devices and their unused channels are exploited to minimize the interference effect on the served device.

\section{B. Imperfect Path Identification}

This subsection quantifies the effect of imperfect D2D path identification on the received SINR at the served $j$ th device. Specifically, based on $s_{I,\left(m_{0}\right), n, j} \geq s_{I,\left(m_{0}-1\right), k, j} \geq$ $\cdots \geq s_{I,(1), n, j}$, for given $\left|\mathcal{M}_{1, j}\right|=m_{0}$, after arranging $\left\{s_{I, m, n, j}\right\}_{m=1}^{m_{0}}$, the device whose index is $m^{\star}$ may be identified to serve the $j$ th user, for $1 \leq m^{\star} \leq m_{0}$. The best scenario is when $m^{\star}=1$ at which $s_{I,\left(m^{\star}\right), n, j}=\min \left\{s_{I, m, n, j}\right\}_{m=1}^{m_{0}}$, whereas the worst scenario occurs when $m^{\star}=m_{0}$, which corresponds to $s_{I,\left(m^{\star}\right), n, j}=\max \left\{s_{I, m, n, j}\right\}_{m=1}^{m_{0}}$. For an arbitrary $m^{\star}$, the resulting observed interference power at the $j$ th device to be served through D2D path can be written as

$$
\begin{aligned}
& \operatorname{Pr}\left\{s_{I,\left(m^{\star}\right), n, j}<x|| \mathcal{M}_{1, j} \mid=m_{0}\right\} \\
& =\sum_{\ell_{1}=m^{\star}}^{m_{0}} \sum_{S_{\ell_{1}}} \prod_{p=1}^{\ell_{1}} \operatorname{Pr}\left\{s_{I, m_{p}, n, j}<x\right\} \\
& \times \prod_{p=\ell_{1}+1}^{m_{0}}\left(1-\operatorname{Pr}\left\{s_{I, m_{p}, n, j}<x\right\}\right) .
\end{aligned}
$$

The sum $S_{\ell_{1}}$ extends over all permutations $\left(m_{1}, m_{2}, \ldots, m_{m_{0}}\right) \quad$ of $1, \ldots, m_{0}$, for which $m_{1}<m_{2}<\cdots<m_{\ell_{1}}$ and $m_{\ell_{1}+1}<\cdots<m_{m_{0}}$. For the special case of identically distributed $\left\{s_{I, m, n, j}\right\}_{m=1}^{m_{0}}$, (19) reduces to

$$
\begin{aligned}
& \operatorname{Pr}\left\{s_{I,\left(m^{\star}\right), n, j}<x|| \mathcal{M}_{1, j} \mid=m_{0}\right\} \\
& =\sum_{\ell_{1}=m^{\star}}^{m_{0}}\left(\begin{array}{c}
m_{0} \\
\ell_{1}
\end{array}\right)\left[\operatorname{Pr}\left\{s_{I, m, n, j}<x\right\}\right]^{\ell_{1}} \\
& \times\left[1-\operatorname{Pr}\left\{s_{I, m, n, j}<x\right\}\right]^{m_{0}-\ell_{1}} .
\end{aligned}
$$

$d t$. Now considering the identification of channel to establish the D2D path to the $j$ th device, and under the effect of imperfect channel identification for an arbitrary $m^{\star}$-indexed device identification, and for given cardinality of the set of unused channels by the $m^{\star}$ th device $\left|\mathcal{N}_{\left(m^{\star}\right)}\right|=n_{\left(m^{\star}\right)}$, define $s_{D,\left(m^{\star}\right),(1), j} \leq s_{D,\left(m^{\star}\right),(2), j} \leq \cdots \leq s_{D,\left(m^{\star}\right),\left(n_{\left(m^{\star}\right)}\right), j}$ that is obtained by arranging $\left\{s_{D,\left(m^{\star}\right), n, j}\right\}_{n=1}^{n_{\left(m^{\star}\right)}}$ in increasing order of magnitude. The best scenario is when $n^{\star}=n_{\left(m^{\star}\right)}$ at which $s_{D,\left(m^{\star}\right),\left(n^{\star}\right), j}=\max \left\{s_{D,\left(m^{\star}\right), n, j}\right\}_{n=1}^{n_{\left(m^{\star}\right)}}$, whereas the worst scenario occurs when $n^{\star}=1$, which corresponds to $s_{D,\left(m^{\star}\right),\left(n^{\star}\right), j}=\min \left\{s_{D,\left(m^{\star}\right), n, j}\right\}_{n=1}^{n_{\left(m^{\star}\right)}}$. Then, for an arbitrary $n^{\star}$, the statistics of the received desired power at the $j$ th device can be expressed as

$$
\begin{aligned}
& \operatorname{Pr}\left\{s_{D,\left(m^{\star}\right),\left(n^{\star}\right), j}<x|| \mathcal{M}_{1, j}\left|=m_{0},\right| \mathcal{N}_{\left(m^{\star}\right)} \mid=n_{\left(m^{\star}\right)}\right\} \\
& =\sum_{\ell_{2}=n^{\star}} \sum_{S_{\ell_{2}}} \prod_{p=1}^{n_{\left(m^{\star}\right)}} \operatorname{Pr}\left\{s_{D,\left(m^{\star}\right), n_{p}, j}<x\right\} \\
& \times \prod_{p=\ell_{2}+1}^{n_{\left(m^{\star}\right)}}\left(1-\operatorname{Pr}\left\{s_{D,\left(m^{\star}\right), n_{p}, j}<x\right\}\right) .
\end{aligned}
$$

The sum $S_{\ell_{2}}$ extends over all permutations $\left(n_{1}, n_{2}, \ldots, n_{n_{\left(m^{\star}\right)}}\right) \quad$ of $1, \ldots, n_{\left(m^{\star}\right)}$, for which $n_{1}<n_{2}<\cdots<n_{\ell_{2}}$ and $n_{\ell_{2}+1}<\cdots<n_{n_{\left(m^{\star}\right)}}$. For 
the special case of identically distributed $\left\{s_{D,\left(m^{\star}\right), n, j}\right\}_{n=1}^{n_{\left(m^{\star}\right)}}$, the result in (21) reduces to

$$
\begin{aligned}
& \operatorname{Pr}\left\{s_{D,\left(m^{\star}\right),\left(n^{\star}\right), j}<x|| \mathcal{M}_{1, j}\left|=m_{0},\right| \mathcal{N}_{\left(m^{\star}\right)} \mid=n_{\left(m^{\star}\right)}\right\} \\
& =\sum_{\ell_{2}=n^{\star}}^{n_{\left(m^{\star}\right)}}\left(\begin{array}{c}
n_{\left(m^{\star}\right)} \\
\ell_{2}
\end{array}\right)\left[\operatorname{Pr}\left\{s_{D,\left(m^{\star}\right), n, j}<x\right\}\right]^{\ell_{2}} \\
& \times\left[1-\operatorname{Pr}\left\{s_{D,\left(m^{\star}\right), n, j}<x\right\}\right]^{n_{\left(m^{\star}\right)}-\ell_{2}} .
\end{aligned}
$$

The received SINR at the $j$ th device for arbitrary identifications of the device and channel indexes can be expressed as

$$
\widetilde{\gamma}_{\mathrm{SINR}, j}=\frac{s_{D,\left(m^{\star}\right),\left(n^{\star}\right), j}}{s_{I,\left(m^{\star}\right),\left(n^{\star}\right), j}+\sigma^{2}},
$$

where the statistics of $s_{I,\left(m^{\star}\right),\left(n^{\star}\right), j}$ and $s_{D,\left(m^{\star}\right),\left(n^{\star}\right), j}$ for given $\left|\mathcal{M}_{1, j}\right|$ and $\left|\mathcal{N}_{\left(m^{\star}\right)}\right|$ are in (19)-(22) for different statistical properties of interference powers and desired powers on possible D2D paths as seen by the $j$ th device, respectively.

The conditional statistics of $\widetilde{\gamma}_{\text {SINR }, j}$ can now be obtained as

$$
\begin{aligned}
& \operatorname{Pr}\left\{\widetilde{\gamma}_{\mathrm{SINR}, j}<x|| \mathcal{M}_{1, j}\left|=m_{0},\right| \mathcal{N}_{\left(m^{\star}\right)} \mid=n_{\left(m^{\star}\right)}\right\} \\
& =\int_{0}^{+\infty} \operatorname{Pr}\left\{s_{D,\left(m^{\star}\right),\left(n^{\star}\right), j}<x\left(t+\sigma^{2}\right)|| \mathcal{M}_{1, j} \mid=m_{0},\right. \\
& \left.\left|\mathcal{N}_{\left(m^{\star}\right)}\right|=n_{\left(m^{\star}\right)}\right\} f_{s_{I,\left(m^{\star}\right),\left(n^{\star}\right), j}}(t) d t .
\end{aligned}
$$

in which the first term in the integrand is given in (21) or (22), and $f_{S_{I,\left(m^{\star}\right),\left(n^{\star}\right), j}}(t)$ defines the distribution of the observed aggregate interference power at the served $j$ th device under imperfect D2D path identification scenarios, which can be obtained as $f_{s_{I,\left(m^{\star}\right),\left(n^{\star}\right), j}}(t) \triangleq \frac{d}{d t}\left[\operatorname{Pr}\left\{s_{I,\left(m^{\star}\right), n, j}<x|| \mathcal{M}_{1, j} \mid=m_{0}\right\}\right]$, with $\operatorname{Pr}\left\{s_{I,\left(m^{\star}\right), n, j}<x|| \mathcal{M}_{1, j} \mid=m_{0}\right\}$ be given in (19) or (20). The unconditional statistics of $\widetilde{\gamma}_{\mathrm{SINR}, j}$ can be then obtained as

$$
\begin{aligned}
& \operatorname{Pr}\left\{\widetilde{\gamma}_{\text {SINR }, j}<x\right\} \\
& =\frac{1}{1-\left[\operatorname{Pr}\left\{\left|\mathcal{M}_{1, j}\right|=0\right\}+\operatorname{Pr}\left\{\left|\mathcal{M}_{1, j}\right|=M\right\}\right]} \\
& \times \sum_{m_{0}=1}^{M-1} \frac{1}{1-\operatorname{Pr}\left\{\left|\mathcal{N}_{\left(m^{\star}\right)}\right|=0|| \mathcal{M}_{1, j} \mid=m_{0}\right\}} \\
& \times \sum_{n_{\left(m^{\star}\right)}=1}^{N-1} \operatorname{Pr}\left\{\widetilde{\gamma}_{\text {SINR }, j}<x|| \mathcal{M}_{1, j}\left|=m_{0},\right| \mathcal{N}_{\left(m^{\star}\right)} \mid=n_{\left(m^{\star}\right)}\right\} \\
& \times \operatorname{Pr}\left\{\left|\mathcal{N}_{\left(m^{\star}\right)}\right|=n_{\left(m^{\star}\right)}|| \mathcal{M}_{1, j} \mid=m_{0}\right\} \operatorname{Pr}\left\{\left|\mathcal{M}_{1, j}\right|=m_{0}\right\}
\end{aligned}
$$

where the conditional statistics of $\widetilde{\gamma}_{\mathrm{SINR}, j}$ in this case can be obtained from (24). Further details are omitted herein due to space limitation.

\section{NumERICAL EXAMPLES}

Some numerical examples on the findings of this paper are presented. It is considered that there are multiple interference sources affecting data link on each physical channel as seen from ready-to-cooperate devices in the set $\mathcal{M}_{1}$. Moreover, transmit powers are given in unit of $\mu W$ and separation

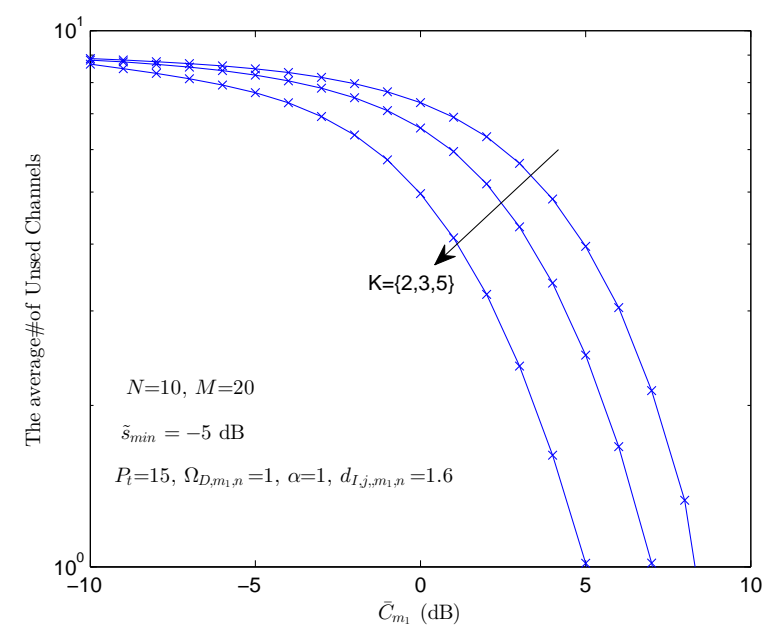

Figure 2. Average number of unused channels by the $m_{1}$ th device whose index belongs to the set $\mathcal{M}_{1}$ and it is fully served by BS versus the device capacity threshold for different number of independent interference sources experienced by that device on each physical channel.

distances between devices and BS as well as among devices in D2D network are given in meters, unless stated otherwise.

Fig. 2 shows the average number of unused channels by the $m_{1}$ th device whose index belongs to $\mathcal{M}_{1}$ and it is fully served by BS versus the device capacity threshold, for different number of independent interference sources experienced by that device on each physical channel. It is noted that when the capacity threshold is very low, the device will likely use only one channel to meet its capacity demand, and hence the average number of unused channels will be approaching its maximum value of $N-1$. This appears for all considered cases of the number of interference sources per each channel as long as the capacity threshold is very low. However, the increase in the capacity threshold of that device demands more channels to be used, wherein the impact of the number of independent interference sources becomes more noticeable. More interference sources per channel leads to less number of unused channels as the device will need to compensate for the increase in the interference effect. The number of unused channels appears to drop sharply as the capacity threshold becomes greater than $1 \mathrm{~b} / \mathrm{s} / \mathrm{Hz}$, where the effect of interference sources grows significantly.

Fig. 3 explains the impact of imperfect D2D path identification on the outage probability of the $j$ th served device in $\mathcal{M}_{2}$ ) through a provisional D2D path. The results compare between the optimal SINR-based identification (shown for comparison purpose) and joint D2D path identification scheme under perfect as well as imperfect identification scenarios. The SINR-based D2D path identification maintains a clear performance advantage over the joint D2D identification under perfect operation. However, this may not hold true under imperfect D2D identification, as when SINR-based identification operates with the worst device-channel pair scenario (case of 


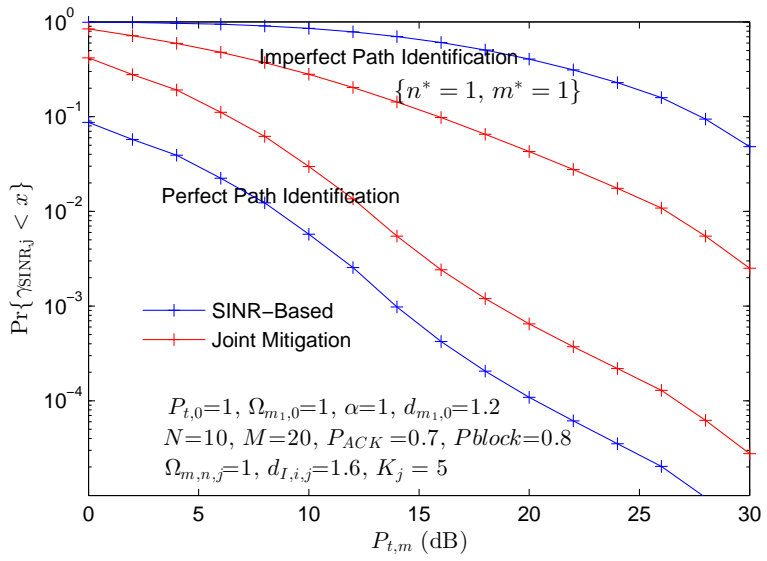

Figure 3. Outage probability of the served device (i.e., the $j$ th device in class $\mathcal{M}_{2}$ ) through provisional D2D path. The results compare between the SINR-based and joint D2D path identification scheme under perfect as well as imperfect identification scenarios.

$n^{\star}=m^{\star}=1$ ). However, for the joint D2D identification under this scenario, the case of $m^{\star}=1$ is exploited to identify the device with the lowest possible interference power effect on the $j$ th served device, while the case of $n^{\star}=1$ represents the worst channel identification scenario. Therefore, under imperfect D2D path identification, the usefulness of the joint D2D path identification can be observed, which can outperform the SINR-based identification.

Fig. 4 discusses further the impact of imperfect joint D2D path identification on the outage probability of the $j$ th served device in $\mathcal{M}_{2}$ ) for different imperfectness scenarios. Compared to the perfect joint D2D path identification scenario when $m^{\star}=1$ and $n^{\star}=\left|\mathcal{N}_{(1)}\right|$, other imperfect scenarios in the figure show degraded outage performance of the $j$ th served device. The amount of performance loss relative to the perfect operation case is directly related to the device and/or channel identification accuracy. Their performance outcomes will be bounded between that of the perfect device and channel identifications and that of simultaneous worst device (case of $\left.m^{\star}=\left|\mathcal{M}_{(1, j)}\right|\right)$ and worst channel (case of $n^{\star}=1$ ) identifications.

\section{Vi. CONCLUSIONS}

This paper has presented new approaches for underlay D2D operation in downlink spectrum-shared heterogeneous cellular networks. It developed detailed explanations for active devices according to their experienced BS coverage, their capabilities to be served by the BS, their abilities to achieve their individual capacity demands, and their needs of spectrum resources. The initiation of D2D cooperation has been conditioned on a provisional spectrum access at an active device. Based on this, D2D path identification scheme has been proposed and quantified for both perfect and imperfect operation conditions. Selected numerical results have showed how the mode of operation and the resulting formulations impact the achieved

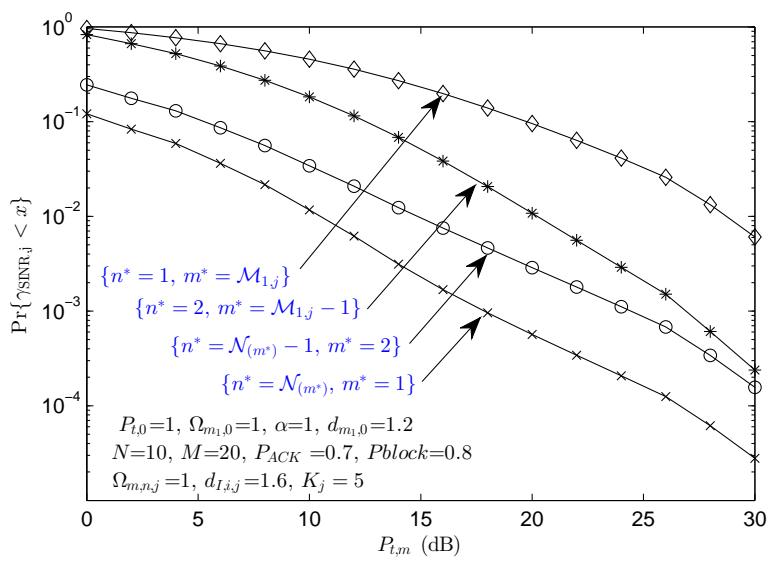

Figure 4. Impact of imperfect joint D2D path identification on the outage probability of the served device (i.e., the $j$ th device in class $\mathcal{M}_{2}$ ) for different imperfectness scenarios.

performance of served device through the proposed provisional D2D path identification.

\section{REFERENCES}

[1] P. Mach, Z. Becvar, and T. Vanek, "In-band device-to-device communication in OFDMA cellular networks: a survey and challenges," IEEE Commun. Surveys Tuts., vol. 17, no. 4, pp. 1885-1919, 2015.

[2] L. Lei, Z. Zhong, C. Lin, and X. Shen, "Operator controlled devicetodevice communications in LTE-advanced networks," IEEE Wireless Commun., vol. 19, no. 3, pp. 96-104, Jun. 2012.

[3] A. Asadi, A. Q. Wang, and V. Mancuso, "A survey on device-to-device communication in cellular networks," IEEE Commun. Surveys Tuts., vol 16, no. 4, pp. 1801-1819, 2014.

[4] Y. Ni, et al., "Beamforming and interference cancellation for D2D communication underlaying cellular networks," IEEE Trans. Commun., vol. 64, no. 2, pp. 832-846, 2016.

[5] H. Wang and X. Chu, "Distance-constrained resource-sharing criteria for device-to-device communications underlaying cellular networks," Electron. Lett., vol. 48, no. 9, pp. 528-530, Apr. 2012.

[6] S. Lv, et al., "Guard zone based interference management for D2D-aided underlaying cellular networks," IEEE Trans. Veh. Technol., vol. 66, no. 6, pp. 5466-5471, 2017.

[7] J. Liu, N. Kato, J. Ma, and N. Kadowaki, "Device-to-device communication in LTE-advanced networks: A survey," IEEE Commun. Surveys Tuts., vol. 17, no. 4, pp. 1923-1940, 2015.

[8] S. Gupta, et al., "Resource allocation for D2D links in the FFR and SFR aided cellular downlink," IEEE Trans. Commun., vol. 64, no. 10, pp. 4434-4448, 2016.

[9] T. Yang, et al., "Graph coloring based resource sharing (GCRS) scheme for D2D communications underlaying full-duplex cellular networks," IEEE Trans. Veh. Technol., vol. 66, no. 8, pp. 7506-7517, 2017.

[10] Z. Uykan and R. Jäntti, "Transmission-order optimization for bidirectional device-to-device (D2D) communications underlaying cellular TDD networks-a Graph theoretic approach," IEEE Jour. Select. Areas Commun., vol. 34, no. 1, pp. 1-14, 2016.

[11] R. M. Radaydeh, F. Gaaloul, and M.-S. Alouini, "Impact of user identities and access conditions on downlink performance in closed small-cell networks," IEEE Trans. Veh. Technol., vol. 65, no. 5, pp. 3200-3216, 2016.

[12] R. M. Radaydeh, et al., "Improved interference-free channel allocation in coordinated multiuser multiantenna open-access small cells," IEEE Trans. Veh. Technol., vol. 65, no. 12, pp. 9994-10010, 2016. 